Article

\title{
PH-Sensitive, Polymer Functionalized, Nonporous Silica Nanoparticles for Quercetin Controlled Release
}

\author{
Lin $\mathrm{Xu}{ }^{1}$, Hong-Liang $\mathrm{Li}^{1, *}$ and Li-Ping Wang ${ }^{2, *(\mathbb{D})}$ \\ 1 College of Materials Science and Engineering, Qingdao University, Qingdao 266071, China; \\ xulinbang@163.com \\ 2 College of Materials Science and Engineering, Liaocheng University, Liaocheng 252059, China \\ * Correspondence: lhl@qdu.edu.cn (H.-L.L.); wangliping5@163.com (L.-P.W.); Tel.: +86-532-8595-0767 \\ (H.-L.L.); +86-635-8230-919 (L.-P.W.)
}

Received: 18 October 2019; Accepted: 4 December 2019; Published: 6 December 2019

\begin{abstract}
Some pH-sensitive, poly(2-(diethylamino)ethyl methacrylate) (PDEAEMA) grafted silica nanoparticles (SNPs) (SNPs-g-PDEAEMA) were designed and synthesized via surface initiated, metal-free, photoinduced atom transfer radical polymerization (ATRP). The structures of the polymers formed in solution were determined by ${ }^{1} \mathrm{H}$ NMR. The modified nanoparticles were characterized by FT-IR spectroscopy, XPS, GPC, TEM and TGA. The analytical results show that $\alpha$-bromoisobutyryl bromide (BIBB) (ATRP initiator) had been successfully anchored onto SNPs' surfaces, and was followed by surface-initiated, metal-free ATRP of 2-(diethylamino)ethyl methacrylate (DEAEMA). The resultant SNPs- $g$-PDEAEMA were uniform spherical nanoparticles with the particles size of about $22-25 \mathrm{~nm}$, and the graft density of PDEAEMA on SNPs' surfaces obtained by TGA was $19.98 \mu \mathrm{mol} / \mathrm{m}^{2}$. Owing to the covalent grafting of $\mathrm{pH}$-sensitive PDEAEMA, SNPs-g-PDEAEMA can dispersed well in acidic aqueous solution, but poorly in neutral and alkaline aqueous solutions, which is conducive to being employed as drug carriers to construct a $\mathrm{pH}$-sensitive controlled drug delivery system. In vitro cytotoxicity evaluation results showed that the cytotoxicity of SNPs- $g$-PDEAEMA to the L929 cells had completely disappeared on the 3rd day. The loading of quercetin on SNPs-g-PDEAEMA was performed using adsorption process from ethanol solutions, and the dialysis release rate increased sharply when the $\mathrm{pH}$ value of phosphate-buffered saline (PBS) decreased from 7.4 to 5.5. All these results indicated that the $\mathrm{pH}$-responsive microcapsules could serve as potential anti-cancer drug carriers.
\end{abstract}

Keywords: silica nanoparticles; PDEAEMA; metal-free ATRP; quercetin; controlled drug release

\section{Introduction}

In recent years, various inorganic-organic hybrid nanomaterials have come out as ideal drug carriers for controlled release systems [1-5]. In this way, inorganic nanomaterials provide a robust framework, while integrated organic components offer functionality. These existing inorganic nanomaterials, such as silica nanoparticles [4,5], $\mathrm{TiO}_{2}$ nanotubes [6] and $\mathrm{ZnO}$ nanoparticles [7], not only conquer the problem of drug delivery, but also improve the therapeutic efficiency of drugs. Silica nanoparticles (SNPs) are considered a carrier of drugs owing to their excellent properties such as large specific surface area, high chemical and mechanical stability, prominent biocompatibility, well-defined surface chemistry and low cytotoxicity [8,9]. Moreover, organic components serve as the "net" of drugs, which can release the encapsulated molecules just as in a specific environment stimulated by the outside world. Meanwhile, $\mathrm{pH}$-sensitive systems have received particular attention in a variety of application stimuli, as it has been shown that the $\mathrm{pH}$ values of tumor tissues (6.5-7.0) are lower those that of bloodstream and normal tissues (7.4), and the pHs of endosomes and lysosomas 
are much lower (5.0-6.5). Therefore, $\mathrm{pH}$-responsive controlled release containers are very necessary in practical applications [10-14].

Surface-initiated atom transfer radical polymerization (SI-ATRP) [15-20] is an important synthetic means for preparing various inorganic-polymer hybrid nanomaterials. However, traditional ATRP requires a transition-metal catalyst (such as $\mathrm{Cu}^{+}$and $\mathrm{Fe}^{2+}$ ) to retain activity throughout the polymerization process, leading to unavoidable metal residues in the final product and impeded applications in some biomedical or electronic fields [21-23]. Photoinduced metal-free ATRP was first reported to solve this drawback in 2014 [24]. In this process, 10-phenylphenothiazine (PTH), an organic-based photoredox catalyst replaced the transition-metal catalyst and is mediated by light; thus, the toxicity of metal catalyst is avoided. Since then, photoinduced, metal-free ATRP has been successfully used as the surface modification of different inorganic materials, such as nanodiamond (ND) [25], SBA-15 [26] and silica [27].

Quercetin $(\mathrm{Qu})$ is the most abundant bioflavonoid with interesting biological properties, such as antiviral, antiallergenic, anti-inflammatory, antibacterial and anticancer activities [8]. The clinical trials have confirmed Qu's effective anticancerous properties and applied it to the treatment of tumor tissues such as those of the colon, liver, brain and prostate to inhibit the spread of malignant cells [28]. Although quercetin is good for the health, its poor bioavailability limits its therapeutic method. The low bioavailability of $\mathrm{Qu}$ is primary due to its restricted absorption capacity (low stability, poor water solubility, short half-life) and its rapid elimination [9].

The primary objective of this study was to design a novel $\mathrm{pH}$-sensitive, inorganic-organic hybrid nanomaterials via photoinduced, metal-free SI-ATRP for Qu controlled release. PDEAEMA was selected to be grafted from SNPs' surfaces for its $\mathrm{pH}$-sensitive and amphiphilic structure; its solubility varies greatly between water soluble and water insoluble corresponding to the protonation and deprotonation of the amine groups on the side depending on the $\mathrm{pH}$ values [12]. Under normal physiological conditions ( $\mathrm{pH}=7.4$ ), the self-assembled Qu-loaded microcapsules have tight and compact structures and the drugs are entrapped in the cores. Meanwhile, in weak acid or neutral conditions, the drug-loaded microcapsules become loose and swollen due to the protonation of the amine groups on PDEAEMA, leading to drug release from the drug-loaded micelles. The detailed reaction and controlled release processes are listed in Scheme 1.

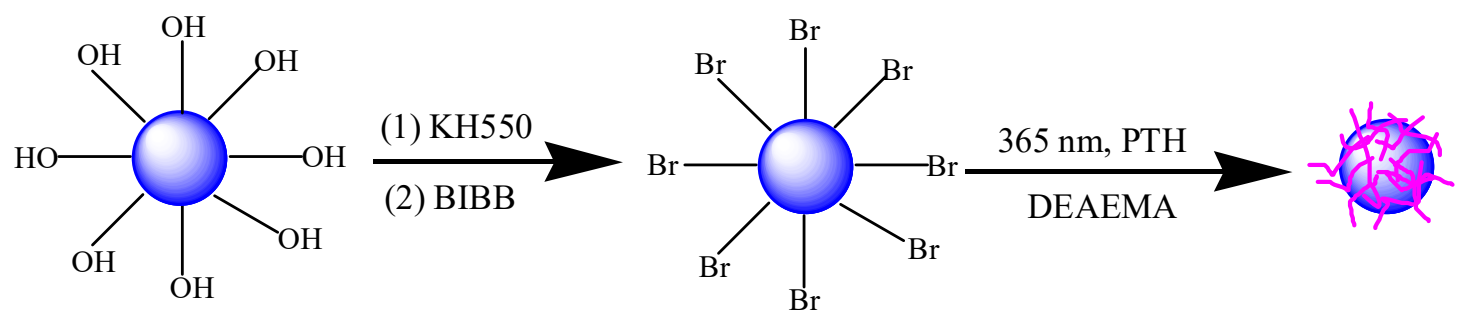

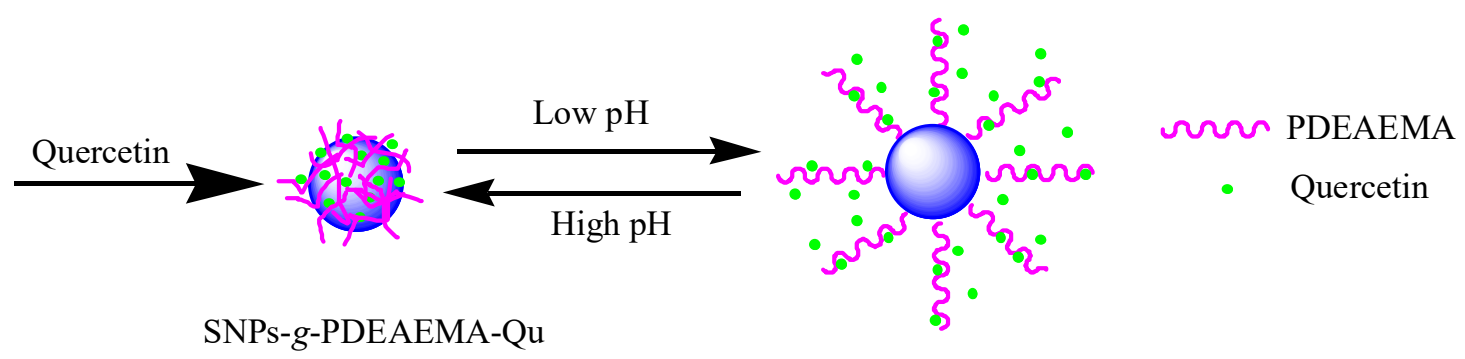

Scheme 1. Synthesis of SNPs- $g$-PDEAEMA, scheme of drug loading and $\mathrm{pH}$-dependent release from SNPs-g-PDEAEMA. 


\section{Experimental Procedures}

\subsection{Synthesis of SNPs-g-PDEAEMA}

SNPs-Br was prepared according to the procedure described in previous study [29]. XPS calculated (atomic \%): Si, 22.05; C, 25.16; N, 5.31; O, 44.22; Br, 3.27; Br, $1.67 \mathrm{mmol}^{-1}$ (calculated according to the $\mathrm{Br}$ atomic \%) (Table 1). SNPs-g-PDEAEMA was synthesized via photoinduced metal-free SI-ATRP. Briefly, DEAEMA ( $2 \mathrm{~mL}, 10 \mathrm{mmol})$, SNPs-Br $(0.15 \mathrm{~g}, 0.250 \mathrm{mmol} \mathrm{Br}$ groups), Ethyl 2-bromoisobutyrate $(5 \mu \mathrm{L}, 0.031 \mathrm{mmol}), \mathrm{PTH}(0.009 \mathrm{~g}, 0.033 \mathrm{mmol}), 1.6 \mathrm{~mL}$ ethanol and $0.4 \mathrm{~mL}$ deionized water were mixed in a $10 \mathrm{~mL}$ quartz tube with a magnetic stirrer. The mixture was stirred for $20 \mathrm{~min}$ at room temperature in the dark. After dispersed uniformly, the reaction mixture was irradiated under a $365 \mathrm{~nm}$ ultraviolet lamp at room temperature in dark for $4 \mathrm{~h}$. The reaction mixture was centrifuged and separated into two parts: a clear liquid at the upper layer (mainly contained the PDEAEMA homopolymer) and precipitation at the bottom (mainly contained crude SNPs-g-PDEAEMA). PDEAEMA homopolymer was obtained by precipitation of the clear liquid in water. In addition, the crude SNPs-g-PDEAEMA was precipitated in water and dried at room temperature under vacuum. Subsequently, the crude SNPs-g-PDEAEMA was purified with ethanol via Soxhlet extraction for $12 \mathrm{~h}$ to get rid of the ungrafted PDEAEMA completely, and dried in vacuum at room temperature. The detailed reaction process is shown in Scheme 1.

Table 1. The element compositions (atomic \%) of SNPs-KH550 and SNPs-Br from XPS results.

\begin{tabular}{c|ccccc}
\hline Elements & O & Si & N & C & Br \\
\hline SNPs-KH550 & 47.09 & 25.75 & 5.77 & 21.40 & 0 \\
SNPs-Br & 44.22 & 22.05 & 5.31 & 25.16 & 3.27 \\
\hline
\end{tabular}

\subsection{Quercetin Loading}

Quercetin loading were performed according to previously described literature [11,30-32]. Typically, quercetin $(0.02 \mathrm{~g})$ and SNPs-g-PDEAEMA $(0.02 \mathrm{~g})$ in weight ratio 1:1 were stirred in ethanol $(1 \mathrm{~mL})$ until the solvent completely evaporated. Then, the powdered products were washed three times with water $(5 \mathrm{~mL})$ to remove the physically absorbed quercetin and dried overnight at $30^{\circ} \mathrm{C}$. The quercetin loaded SNPs-g-PDEAEMA formulation was designated as SNPs-g-PDEAEMA-Qu. The amount of loaded quercetin for SNPs-g-PDEAEMA was monitored by a fluorescence spectrophotometer at $367 \mathrm{~nm}$. The drug concentration was calculated according to the standard curve of pure quercetin dissolved in ethanol. The drug loading capacity (33.82\%) and entrapment efficiency (67.64\%) were calculated using the following formula.

$$
\begin{gathered}
\text { Drug loading }(\%)=\frac{\text { weight of drug in SNPs }-g-\text { PDEAEMA }}{\text { weight of SNPs }-g-\text { PDEAEMA }- \text { Qu }} \times 100 \% \\
\text { Entrapment efficiency }(\%)=\frac{\text { weight of drug in SNPs }-g-\text { PDEAEMA }}{\text { initial weight of drug }} \times 100 \%
\end{gathered}
$$

\subsection{In Vitro Cytotoxicity Evaluation}

According to ISO 10993-5 Standard and previously literature [33-35], the in vitro cytotoxicity of SNPs-g-PDEAEMA was evaluated on an extracted solution of SNPs- $g$-PDEAEMA via MTT assay. In Briefly, the sterilized SNPs-g-PDEAEMA was extracted using DMEM at an extraction ratio of $0.5 \mathrm{mg} \cdot \mathrm{mL}^{-1}$ for $24 \mathrm{~h}$ at $37^{\circ} \mathrm{C}$. The medium $(100 \mu \mathrm{L})$ containing $10^{4} \mathrm{~L} 929$ cells and extract solution $(100$ $\mu \mathrm{L}$ ) was plated into each well of the 96-well plate and then incubated in $5 \% \mathrm{CO}_{2}$ atmosphere at $37^{\circ} \mathrm{C}$. MTT ( $20 \mu \mathrm{L}, 5 \mathrm{mg} \cdot \mathrm{mL}^{-1}$ in PBS) was added for $4 \mathrm{~h}$ and allowing the formation of formazan crystals on the 1st, 2nd and 3rd days. After removing the supernatant, $150 \mu \mathrm{L}$ DMSO was added to each well, 
and the absorbance was monitored by a microplate reader at $490 \mathrm{~nm}$. The data were expressed as percentages relative to the data obtained with blank control. Each group was tested with six samples.

\subsection{Cell Uptake Assay}

L929 cells were inoculated on the focusing plate with a cellular concentration of $1 \times 10^{5}$, and incubated for $24 \mathrm{~h}$. In total, $10 \mathrm{ug} / \mathrm{ml}$ of cy5.5-labeled SNPs-g-PDEAEMA-Qu (SNPs-g-PDEAEMAQu-cy5.5) was added, and the resulting solution was incubated for 0.5, 2 and $4 \mathrm{~h}$. Cell nucleus were stained with blue fluorescence by Hoechst 33258. Cell uptake images were recorded using an ix-71 inverted fluorescence microscope (Olympus, Japan).

\subsection{In Vitro Release of Quercetin from SNPs-g-PDEAEMA}

In vitro quercetin drug release research was carried out in buffer solution with $\mathrm{pH}$ values of 5.5 and 7.4 at $37^{\circ} \mathrm{C}$. In a typical experiment, $2.0 \mathrm{mg}$ of SNPs-g-PDEAEMA-Qu was suspended in $3.0 \mathrm{~mL}$ of phosphate-buffered saline (PBS) ( $\mathrm{pH} 5.5$ or 7.4). The resulting solution was moved to a dialysis bag (molecular weight cutoff 7500-8000), placed in conical flasks containing $97 \mathrm{~mL}$ of PBS and vibrated at thermostatic water bath oscillator. At appropriate time interval, $3 \mathrm{~mL}$ solution samples $\left(V_{\mathrm{e}}\right)$ were removed from the release medium and an equal volume of fresh buffer was added to maintain the total volume. The release of quercetin in different PBS was monitored via UV-Vis spectroscopy at the wavelength of $367 \mathrm{~nm}$. The cumulative percentage of drug release $\left(E_{\mathrm{r}}\right)$ was calculated according to the calibration curve $(r>0.999)$ and the following Equation (3).

$$
\operatorname{Er}(\%)=\frac{V_{e} \sum_{1}^{n-1} C_{i}+V_{0} C_{n}}{m_{Q_{u}}} \times 100 \%
$$

where $m_{Q u}$ is the amount of quercetin in the SNPs-g-PDEAEMA, $\mathrm{Cn}$ is the concentration of quercetin in the $n_{\text {th }}$ sample, $V_{0}$ represents the volume of the release medium $\left(V_{0}=100 \mathrm{~mL}\right)$. The in vitro release of quercetin was performed in triplicate at different $\mathrm{pH}$ conditions to gain the final in vitro release curves.

\section{Results and Discussion}

\subsection{Material Characterization}

\subsubsection{Structure Characterization}

The immobilization ATRP initiator onto SNPs' surfaces is necessary for prepare the polymer brushes grafted from SNPs' surfaces by metal-free SI-ATRP. The chemical compositions of SNPs-KH550 and SNPs-Br were measured by XPS analysis. Figure 1a shows the wide-scan XPS spectra of SNPs-KH550. The signals of N1s $(398.7 \mathrm{eV})$ and $\mathrm{C}_{1 \mathrm{~s}}(281.5 \mathrm{eV})$ were attributed to the $\mathrm{N}$ and C of KH550 on SNPs surface. The still-observed peaks for $\mathrm{Si}_{2 \mathrm{p}}(101.2 \mathrm{eV})$ were ascribed to the exposed Si element of SNPs. As for SNPs-Br (Figure 1b), except for the main signals of O 1s, N 1s, C 1s and Si 2p, were still observed at 533.6, 400.2, 283.3 and $102.8 \mathrm{eV}$ (Figure 1b-f); a new signal of Br element appeared at $69.3 \mathrm{eV}$ (Figure $1 \mathrm{~b}, \mathrm{~g}$ ). In addition, the N 1s core-level spectra of SNPs-Br showed two peak components at $399.3 \mathrm{eV}$ and $401.0 \mathrm{eV}$ (Figure 1d). The peak at $399.3 \mathrm{eV}$ was ascribed to $\mathrm{N}-\mathrm{C}\left(\mathrm{sp}^{3}\right)$ and the peak at the $401.0 \mathrm{eV}$ corresponds to $\mathrm{N}-\mathrm{C}=\mathrm{O}$ bonds. The $\mathrm{C} 1 \mathrm{~s}$ core-level spectra of SNPs-Br consisted with five types bond, including $\mathrm{C}-\mathrm{C}, \mathrm{C}-\mathrm{H}, \mathrm{C}-\mathrm{N}, \mathrm{C}=\mathrm{O}$ and $\mathrm{C}-\mathrm{Br}$, accompanied by the appearance of peaks at $284.8,284.2,286.4,288.1$ and $285.6 \mathrm{eV}$, respectively (Figure 1f). These results indicated successful immobilizing of BIBB initiators onto SNPs' surfaces. The existence of $\mathrm{Br}$ provided the prerequisite conditions for subsequent metal-free SI-ATRP. 

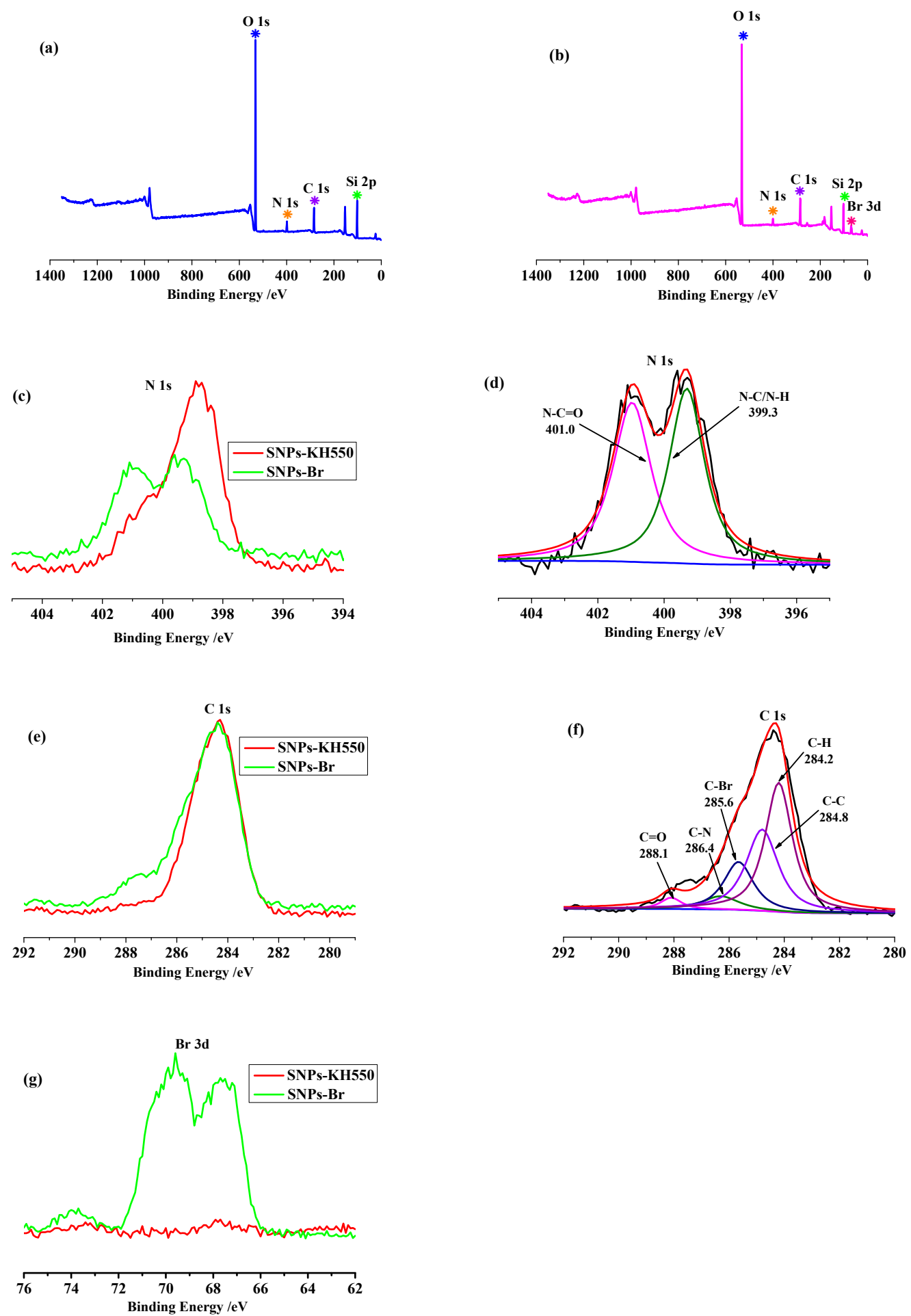

Figure 1. XPS wide-scan spectra of silica nanoparticles (SNPs)-KH550 (a) and SNPs-Br (b). XPS high resolution spectra of (c) N1s of SNPs-KH550 and SNPs-Br, (d) N1s core-level spectra of SNPs-Br, (e) C1s of SNPs-KH550 and SNPs-Br, (f) C1s core-level spectra of SNPs-Br and (g) Br3d of SNPs-KH550 and SNPs-Br. 
The FT-IR spectra of SNPs, SNPs-KH550, SNPs-Br and SNPs-g-PDEAEMA are shown in Figure 2. The adsorption peaks at $1093 \mathrm{~cm}^{-1}$ were ascribed to the $\mathrm{Si}-\mathrm{O}-\mathrm{Si}$ stretching vibration of SNPs. Compared with crude SNPs, the FT-IR spectra of SNPs-KH550 (Figure 2b) shows a new characteristic peak at $2930 \mathrm{~cm}^{-1}$ which was attributed to $\mathrm{CH}_{2}$ stretching vibration of $\mathrm{KH} 550$ on the SNP surface. As for the SNPs-Br (Figure 2c), a new characteristic peak appeared at $1731 \mathrm{~cm}^{-1}$, which can be assigned to the stretching vibration of $\mathrm{C}=\mathrm{O}$ in $\mathrm{BiBB}$, indicating the successful amination of acyl bromide groups and amino groups. After metal free SI-ATRP (Figure 2d), the greatly enhancive peak at $1731 \mathrm{~cm}^{-1}$ was attributed to the stretching vibration of $\mathrm{C}=\mathrm{O}$, which is the result of the $\mathrm{C}=\mathrm{O}$ overlap of $\mathrm{BiBB}$ and PDEAEMA. The FT-IR results illustrate the successful grafting of PDEAEMA from SNPs surface via metal-free SI-ATRP. In addition, the molecular weight and distribution of the cleaved PDEAEMA from SNPs surface determined by GPC further supported the presence of PDEAEMA grafted from SNPs surface (see Table S1).

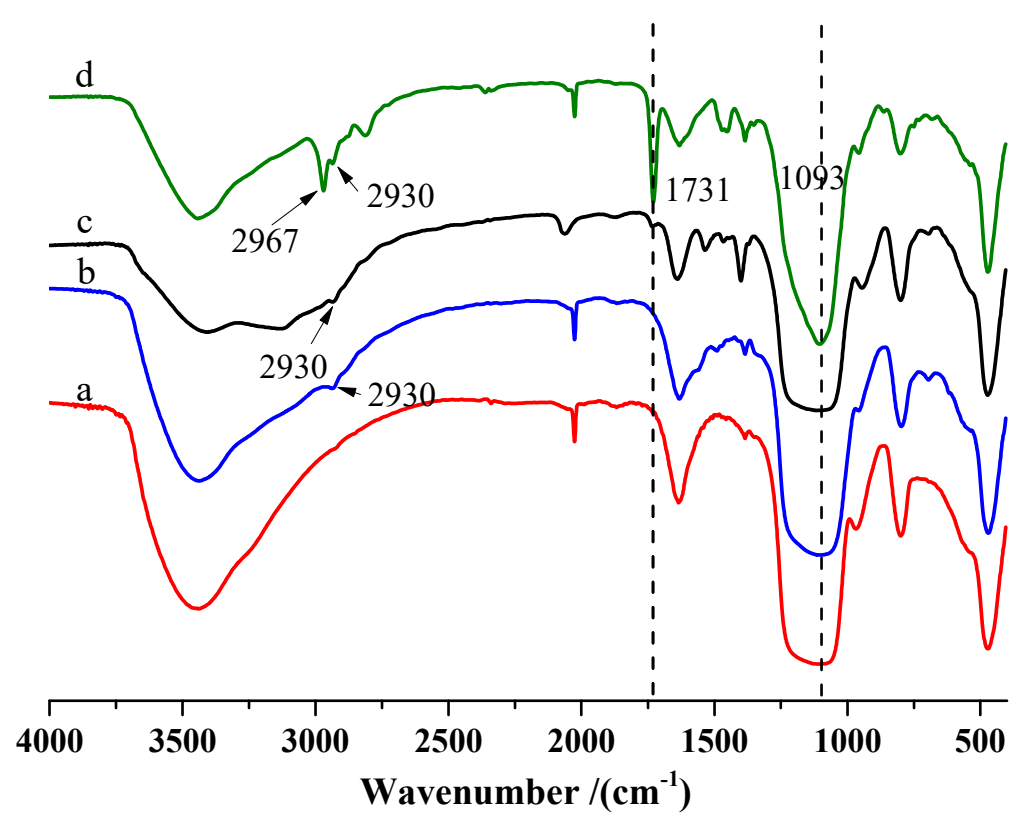

Figure 2. FT-IR spectra of (a) SNPs, (b) SNPs-KH550, (c) SNPs-Br and (d) SNPs- $g$-PDEAEMA.

As a significant analytical means, the ${ }^{1} \mathrm{HNMR}$ spectrum can adequately analyze the structure of PDEAEMA formed in solution (Figure 3). The signal at $0.00 \mathrm{ppm}$ belongs to the NMR internal reference (TMS). The signal at $4.00 \mathrm{ppm}$ was ascribed to the proton peak of $-\mathrm{OCH}_{2}$, and $2.72 \mathrm{ppm}$ was the proton peak of $\mathrm{CH}_{2}$ connected with $\mathrm{OCH}_{2}$ in PDEAEMA. The signal at $2.58 \mathrm{ppm}$ was assigned to the proton peak of $\mathrm{NCH}_{2}$ connected with the branch chain, and $1.04 \mathrm{ppm}$ was due to the proton peak of $\mathrm{CH}_{3}$ connected with $\mathrm{NCH}_{2}$. The signals at 0.90 and $1.81 \mathrm{ppm}$ were attributed to the repeating units of methyl $\left(\mathrm{CH}_{3}\right)$ and methylene $\left(\mathrm{CH}_{2}\right)$ in the main polymer chain. The $\mathrm{H}^{1} \mathrm{NMR}$ results indicate that metal free SI-ATRP reaction was successful. 


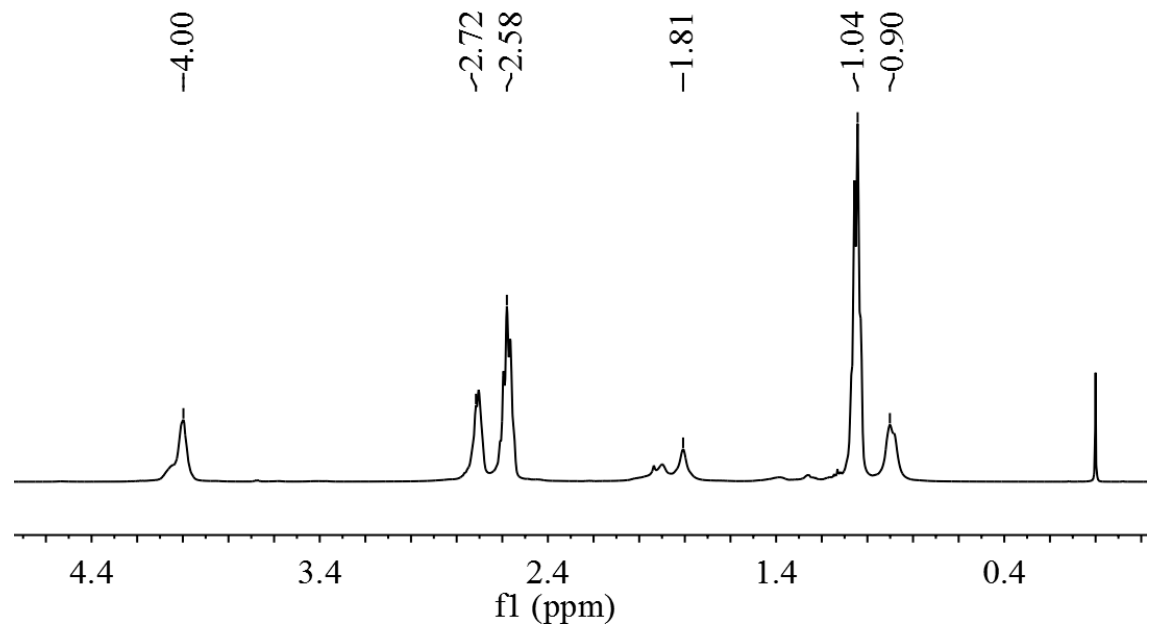

Figure $3 .{ }^{1} \mathrm{H}$ NMR spectrum of PDEAEMA formed in solution.

\subsubsection{Morphology Analysis}

To get more information about the effect of PDEAEMA grafting from the SNPs surface, TEM and Nano Measurer software were used to investigating the surface topography and particle size and distribution in detail. Figure 4 presents the TEM images of SNPs and SNPs- $g$-PDEAEMA. It can be seen that the particles' sizes exhibit a tendency of increasing with the process of grafting polymerization. The particle sizes of pure SNPs are mainly concentrated at $13-18 \mathrm{~nm}$. The smaller particle size of the particles owe themselves to the higher specific surface energy, which makes them tend to aggregate and difficult to disperse (Figure 4a). This phenomenon impeded the application and development of SNPs. But after grafting PDEAEMA from SNPs surface by metal-free ATRP (Figure $4 \mathrm{~b}$ ), the particle size of SNPs-g-PDEAEMA increased (it was mainly distributed at 22-25 nm) and the dispersion degree was also improved. This is due to the amphiphilic PDEAEMA grafted from SNPs surfaces' allowing them to interact with each other to promote dispersion.

(a)

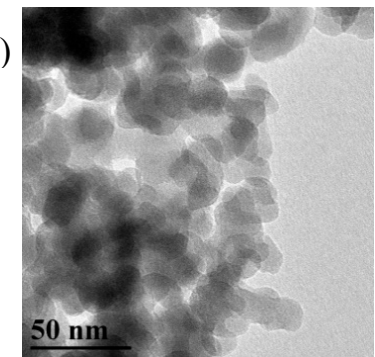

(b)

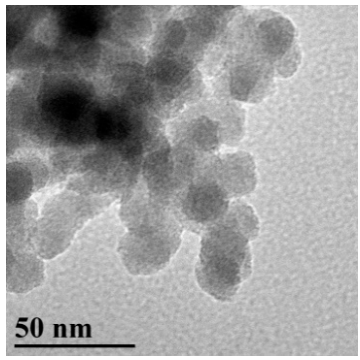

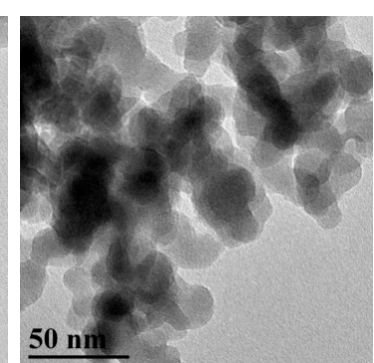

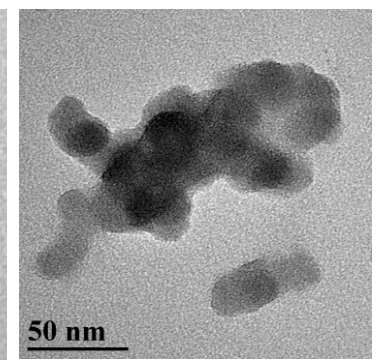

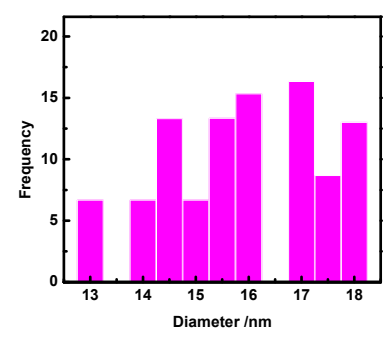

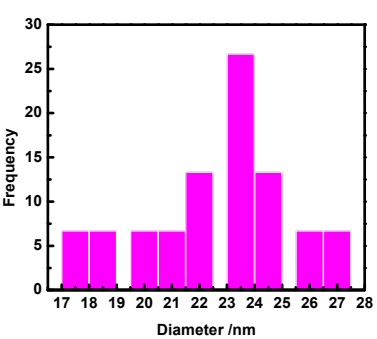

Figure 4. TEM images of (a) SNPs, (b) SNPs-g-PDEAEMA and the particle size distribution.

\subsubsection{TGA and DSC Analysis}

The grafting percentage of PDEAEMA from SNPs surface and the thermostability of the samples were determined by TGA. Figure 5 exhibits the TGA curves of SNPs, SNPs-KH550, SNPs-Br and 
SNPs- $g$-PDEAEMA with the temperature rising from 25 to $800{ }^{\circ} \mathrm{C}$. The weight losses of SNPs, SNPs-KH550, SNPs-Br and SNPs- $g$-PDEAEMA were $6.80 \%, 15.20 \%, 22.38 \%$ and $44.86 \%$, respectively. The graft density $\mathrm{G}_{\varphi}$ in $\mu \mathrm{mol} / \mathrm{m}^{2}$ and molecules $/ \mathrm{nm}^{2}$, and the percent of functionalization or level of surface coverage (f), for all functionalized materials, can be calculated via the weight loss using Equation 4 reported by Bonilla-Cruz et al [36]. The values obtained are listed in Table 2. That is, the graft density of PDEAEMA on SNPs surface is $19.98 \mu \mathrm{mol} / \mathrm{m}^{2}$. Figure S1 shows DSC curves of PDMAEMA and SNPs- $g$-PDMAEMA. As can be seen in Figure S1, the experimental values of $T_{\mathrm{g}}$ obtained for PDEAEMA and SNPs- $g$-PDMAEMA were 22.5 and $31.5^{\circ} \mathrm{C}$ respectively. The increased $T_{\mathrm{g}}$ of PDMAEMA in SNPs- $g$-PDMAEMA, compared to pure PDMAEMA, probably caused by the confinement effect of SNPs leading to the inhibition of the chain movement of PDEAEMA.

$$
\mathrm{G} \varphi_{1}\left(\frac{\mu \mathrm{mol}}{\mathrm{m}^{2}}\right)=\frac{\frac{\mathrm{W}_{60-800}}{100-\mathrm{W}_{60-800}} \times 100-\mathrm{W}_{\mathrm{SNPs}}}{\mathrm{M}_{\mathrm{n}} \times \mathrm{S}_{\mathrm{spec}} \times 100} \times 10^{6}
$$

where $\mathrm{W}_{\mathrm{SNPs}}$ is the weight loss of SNPs (dihydroxylation) before functionalization, and $\mathrm{W}_{60-800}$ is the weight loss between 60 and $800{ }^{\circ} \mathrm{C}$ corresponding to the thermal decomposition of KH550, BIBB and PDEAEMA respectively. $\mathrm{Mn}$ is the molecular weight of the degradable part of each of the grafted KH550, BIBB and PDEAEMA, respectively. $S_{\text {spec }}$ is the specific surface area of an SNP.

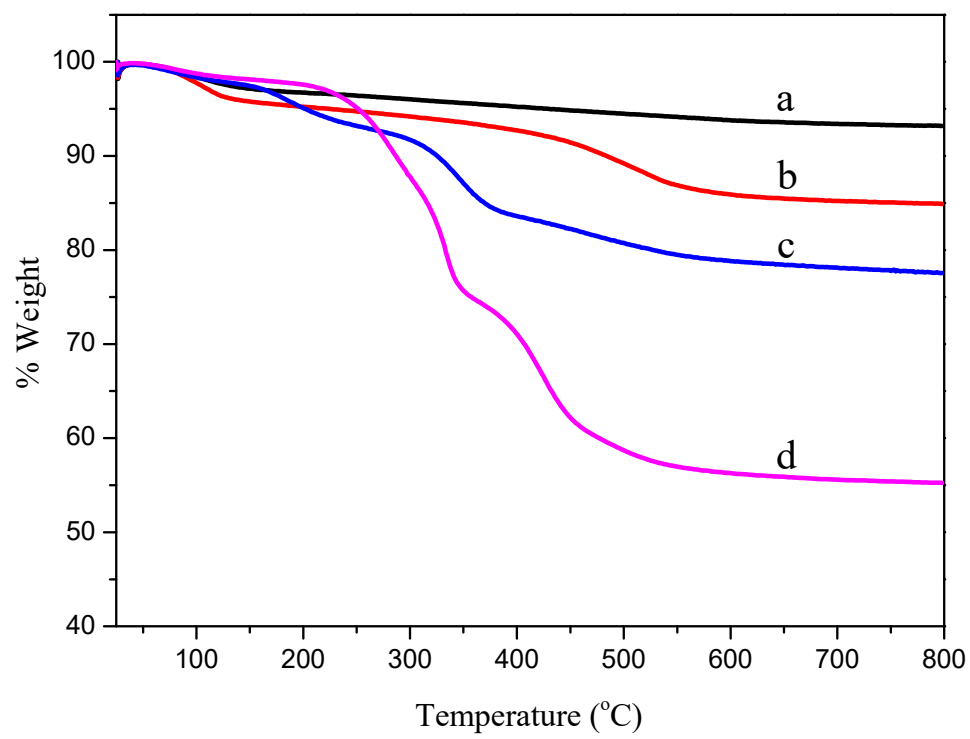

Figure 5. TGA curves of SNPs (a), SNPs-KH550 (b), SNPs-Br (c) and SNPs- $g$-PDEAEMA (d).

Table 2. The TGA analysis of SNPs grafted with different organics.

\begin{tabular}{|c|c|c|c|}
\hline Sample & ${ }^{\mathrm{a}} \mathrm{G}_{\varphi 1}\left(\mu \mathrm{mol} / \mathrm{m}^{2}\right)$ & ${ }^{b} G_{\varphi 2}\left(\right.$ molecules $\left./ \mathrm{nm}^{2}\right)$ & ${ }^{c} f(\%)$ \\
\hline SNPs-KH550 & 3.05 & 1.84 & 62.79 \\
\hline SNPs-Br & 3.59 & 2.17 & 74.06 \\
\hline SNPs- $g$-PDEAEMA & 19.98 & 12.03 & 419.79 \\
\hline
\end{tabular}
$\times 100$.

\subsubsection{Dispersibility and pH-Responsive Properties Analysis}

Figure 6 exhibits the images of the dispersions of SNPs- $g$-PDEAEMA in acid, neuter and alkaline aqueous solutions. It can be observed, obviously, that the hybrid materials are dispersed in acid but gathered in neuter and alkaline aqueous solutions. This is because PDEAEMA contains tertiary amine group, which is a weak base. Under acidic conditions, tertiary amine groups are protonized, and the 
polymer chain stretches due to mutual repulsion between electric charges. Under neutral and alkaline conditions, the protonation of groups is weakened or disappeared, the mutual repulsion between the charges on the polymer chains is weakened and the attraction between the polymers is enhanced, so the polymer chains curl and contract in water. This result further illustrated that the $\mathrm{pH}$-responsive monomer DEAEMA successfully grafted from SNPs surface.

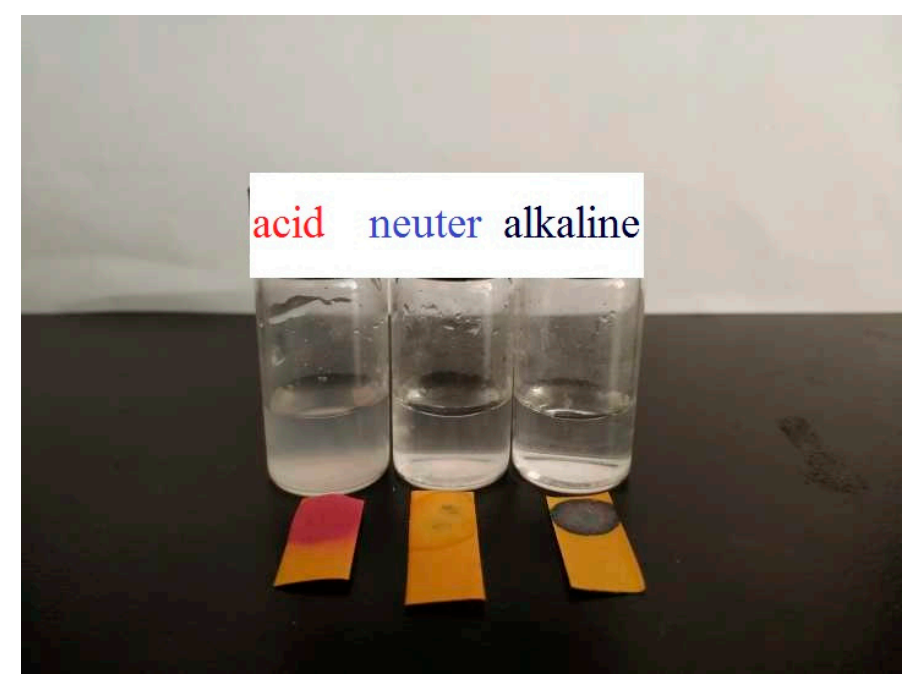

Figure 6. The dispersibility of SNPs-g-PDEAEMA in acid, neuter and alkaline aqueous solutions, respectively.

\subsection{In Vitro Cytotoxicity Evaluation and Cell Uptake Assay}

The in vitro cytotoxicity of SNPs- $g$-PDEAEMA and a control sample were evaluated by MTT assay using L929 cells incubated with the extracted solution of SNPs-g-PDEAEMA for 1, 2 and 3 days. The results of quantitative evaluation of the cytotoxicity are shown in Figure 7. As shown in Figure 7, on the 1st and 2nd day, the cell viabilities were $90.18 \%$ and $92.43 \%$, respectively; on the 3rd day, the cytotoxicity of SNPs-g-PDEAEMA to the L929 cells had completely disappeared, which can be ascribed to the biocompatible nature of SNPs- $g$-PDEAEMA. The value of the relative growth rate (RGR), confirmed the biocompatibility of the SNPs-g-PDEAEMA, sufficiently meets the requirements of the subsequent biomedical applications.

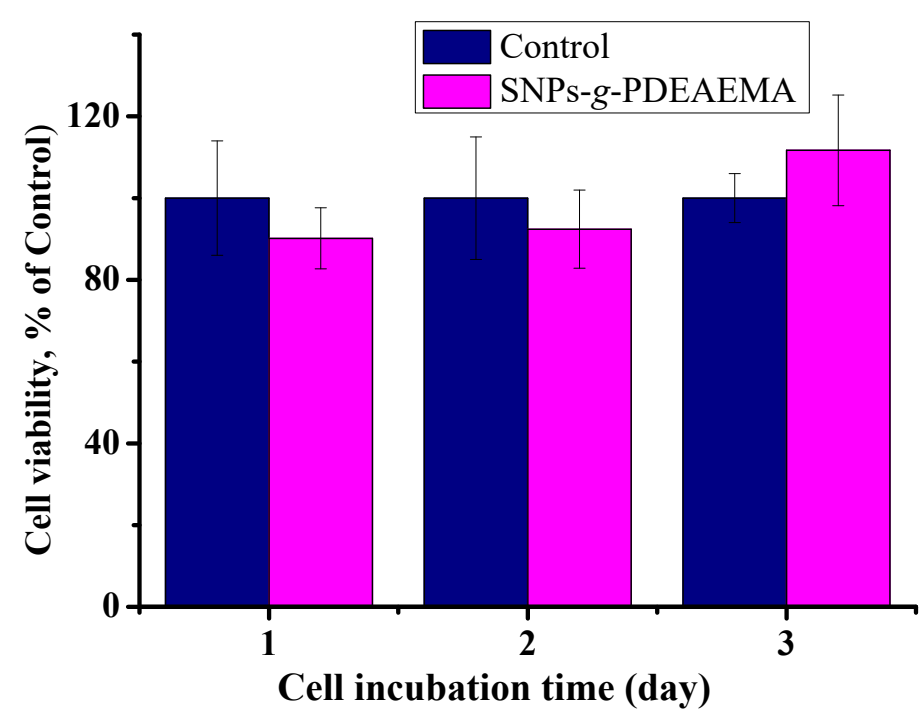

Figure 7. In vitro cytotoxicity of control sample and SNPs- $g$-PDEAEMA towards L929 cells. 
To observe the cell uptake of SNPs-g-PDEAEMA-Qu, a fluorescence microscope (FM) was employed to track the red fluorescence of SNPs-g-PDEAEMA-Qu (labeled by Cy5.5) in L929 cells (Figure 8). As can be seen from Figure 8, cy5.5-labeled SNPs-g-PDEAEMA-Qu (SNPs-g-PDEAEMA-Qu-cy5.5) showed red fluorescence, cell nuclei exhibited blue fluorescence after staining by Hoechst 33258 and SNPs-g-PDEAEMA-Qu-cy5.5 was successfully absorbed into the cytoplasm around the cell nucleus. Moreover, the uptake is time-dependent and increases with the extension of time, indicating that SNPs- $g$-PDEAEMA as a drug carrier can be endocytosis by cells; thus, promoting drug absorption.

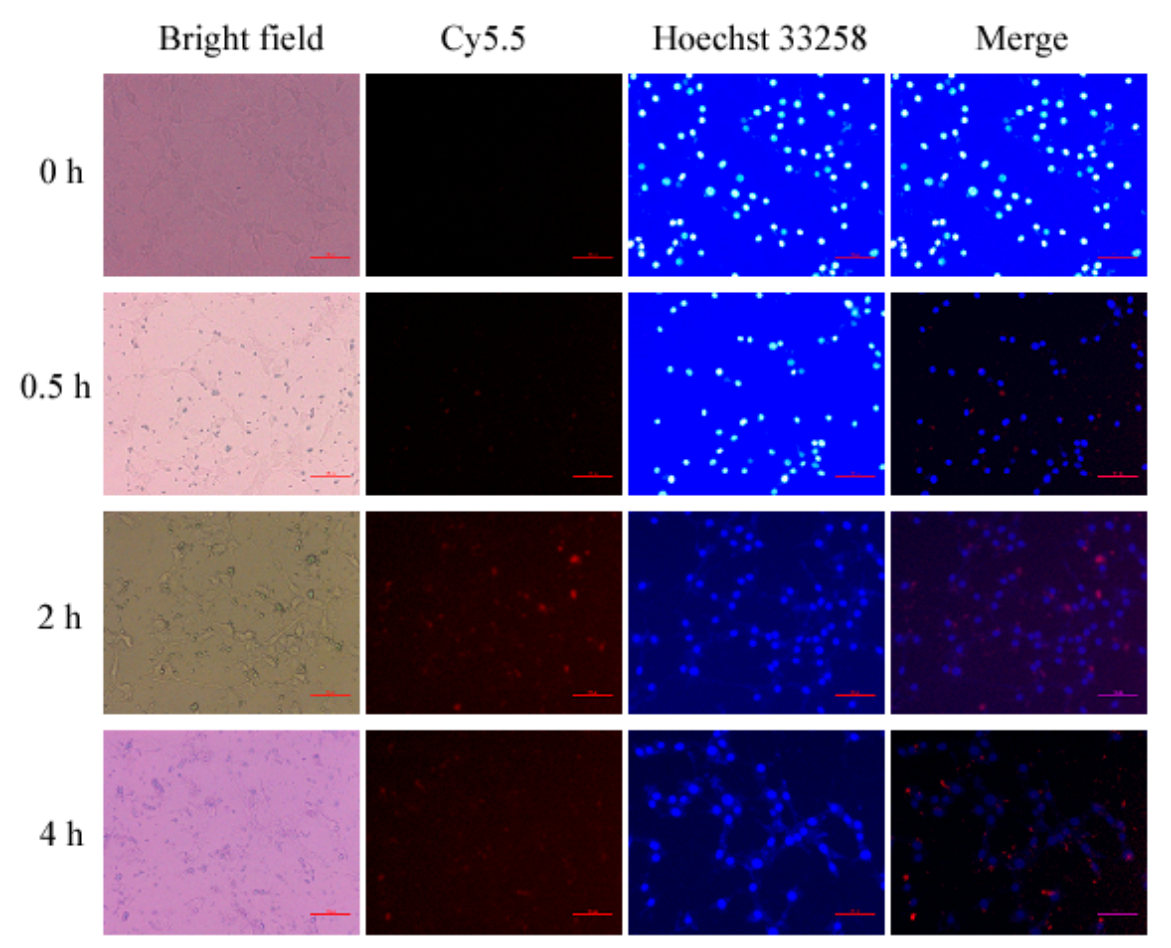

Figure 8. Fluorescence microscope images of L929 cells treated with SNPs-g-PDEAEMA-Qu-cy5.5 for $0.5,2$ and $8 \mathrm{~h}$, while non-treated cells were used as the control $(0 \mathrm{~h})$.

\subsection{Quercetin Loading and In Vitro Release}

In order to assess the effect of $\mathrm{pH}$-sensitive properties on controlled drug release, in vitro quercetin release behavior of SNPs- $g$-PDEAEMA-Qu was measured under in a slightly acidic environment (PBS, pH 5.5) and physiological conditions (PBS, pH 7.4). Figure 9 shows the drug release curve of SNPs-g-PDEAEMA-Qu in buffer solution (PBS, pH 5.5 or $7.4,37^{\circ} \mathrm{C}$ ). It can be observed that the release amount significantly sped up when the $\mathrm{pH}$ value decreased from 7.4 to 5.5. At $\mathrm{pH} 7.4$, about $33 \%$ of quercetin was released in vivo within $108 \mathrm{~h}$, which indicates that the drug can be well protected and stays stable with minimal quercetin release from SNPs- $g$-PDEAEMA at normal physiological conditions. At $\mathrm{pH} 5.5$, about $67 \%$ of the quercetin is released at $108 \mathrm{~h}$. Therefore, the release of quercetin is $\mathrm{pH}$-responsive; when the $\mathrm{pH}$ value decreased from 7.4 to 5.5, protonated PDEAEMA chains repulsed each other electrostatically and extended, accelerating the release behavior of quercetin efficiently. An acidic environment can simulate the environment of tumor tissue or the human stomach, while an alkaline one can simulate the environment of normal tissues or the large intestine. Therefore, $\mathrm{pH}$-responsive SNPs- $\mathrm{g}$-PDEAEMA is expected to be used in clinical trials to control drug release behavior. 


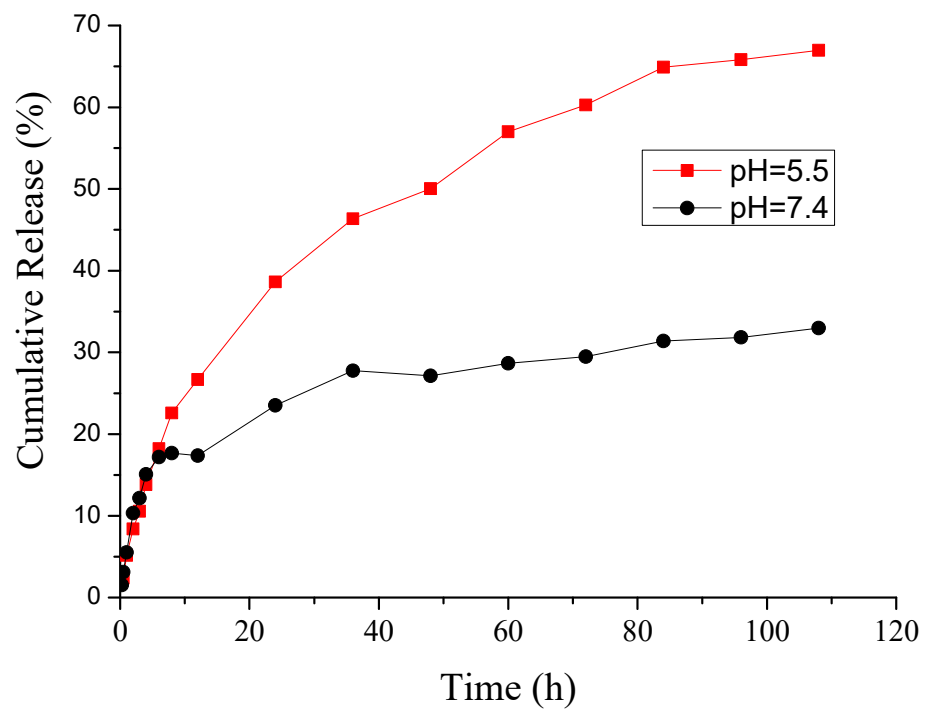

Figure 9. In vitro quercetin release curves of SNPs- $g$-PDEAEMA-Qu at different $\mathrm{pH}$ values.

\section{Conclusions}

In summary, $\mathrm{pH}$-responsive SNPs-g-PDEAEMA via metal free SI-ATRP has been successfully synthesized. The $\alpha$-bromoisobutyryl bromide (BIBB) (ATRP initiator) was immobilized onto SNPs' surfaces to initiate metal-free photoinduced SI-ATRP of DEAEMA. The morphology and chemical composition of the SNPs- $g$-PDEAEMA were verified well by TEM, FT-IR, ${ }^{1} \mathrm{HNMR}$, GPC and TGA. These results demonstrated that PDEAEMA is successfully grafted from the SNPs surfaces. For its $\mathrm{pH}$-responsive properties, $\mathrm{Qu}$ is loaded into SNPs-g-PDEAEMA via an adsorption process from ethanol solutions. The in vitro release displayed $\mathrm{pH}$ dependence, showing a significant increase in rate as the $\mathrm{pH}$ value decreased from 7.4 to 5.5. Acidic conditions can simulate the environment of tumor tissues, while alkaline conditions can simulate the environment of normal tissues. Therefore, the $\mathrm{pH}$-responsive SNPs- $g$-PDEAEMA is expected to be used in clinical trials to control drug release behavior.

Supplementary Materials: The supplementary materials are available online at http://www.mdpi.com/2073-4360/ 11/12/2026/s1.

Author Contributions: L.X., and L.-P.W. performed the experiments, analyzed the data and wrote the paper; H.-L.L. and L.-P.W. conceived and designed the experiments and revised the paper.

Funding: This research was funded by the financial support from the Double First Class University Construction of Shandong Province and the Taishan Scholars Advantageous and Distinctive Discipline Program of Shandong Province for supporting the research team of energy storage materials, the Shandong province higher education science and technology program (J16LA01), the Natural Science Foundation of China (number 51603098).

Conflicts of Interest: The authors declare no conflict of interest.

\section{References}

1. Singh, R.K.; Patel, K.D.; Mahapatra, C.; Parthiban, S.P.; Kim, T.H.; Kim, H.W. Combinatory Cancer Therapeutics with Nanoceria-Capped Mesoporous Silica Nanocarriers through pH-triggered Drug Release and Redox Activity. ACS Appl. Mater. Interfaces 2019, 11, 288-299. [CrossRef]

2. Skwira, A.; Szewczyk, A.; Prokopowicz, M. The Effect of Polydimethylsiloxane-EthylcelluloseCoating Blends on the Surface Characterizationand Drug Release of Ciprofloxacin-Loaded Mesoporous Silica. Polymers 2019, 11, 1450. [CrossRef]

3. Bulbul, Y.E.; Eskitoros-Togay, S.M.; Demirtas-Korkmaz, F.; Dilsiz, N. Multi-walled carbon nanotubeincorporating electrospun composite fibrous mats for controlled drug release profile. Int. J. Pharm. 2019, 568, 118513. [CrossRef] [PubMed] 
4. Tian, B.; Liu, S.; Wu, S.; Lu, W.; Wang, D.; Jin, L.; Hu, B.; Li, K.; Wang, Z.; Quan, Z. pH-responsive poly(acrylic acid)-gated mesoporous silica and its application in oral colon targeted drug delivery for doxorubicin. Colloids Surf. B 2017, 154, 287-296. [CrossRef] [PubMed]

5. Li, X.; Wang, X.; Hua, M.; Yu, H.; Wei, S.; Wang, A.; Zhou, J. Photothermal-Triggered Controlled Drug Release from Mesoporous Silica Nanoparticles Based on Base-Pairing Rules. ACS Biomater. Sci. Eng. 2019, 5, 2399-2408. [CrossRef]

6. Mohan, L.; Anandan, C.; Rajendran, N. Drug release characteristics of quercetin-loaded $\mathrm{TiO}_{2}$ nanotubes Coated with chitosan. Int. J. Biol. Macromol. 2016, 93, 1633-1638. [CrossRef]

7. Ensafi, A.A.; Khoddami, E.; Nabiyan, A.; Rezaei, B. Study the role of poly(diethyl aminoethyl methacrylate) as a modified and grafted shell for $\mathrm{TiO}_{2}$ and $\mathrm{ZnO}$ nanoparticles, application in flutamide delivery. React. Funct. Polym. 2017, 116, 1-8. [CrossRef]

8. Vergara-Castañeda, H.; Hernandez-Martinez, A.R.; Estevez, M.; Mendoza, S.; Luna-Barcenas, G.; Pool, H. Quercetin conjugated silica particles as novel biofunctional hybrid materials for biological applications. J. Colloid Interface Sci. 2016, 466, 44-55. [CrossRef]

9. Sarkar, A.; Ghosh, S.; Chowdhury, S.; Pandey, B.; Sil, P.C. Targeted delivery of quercetin loaded mesoporous silica nanoparticles to the breast cancer cells. Biochim. Biophys. Acta 2016, 1860, 2065-2075. [CrossRef]

10. Chang, Y.J.; Liu, X.Z.; Zhao, Q.; Yang, X.H.; Wang, K.M.; Wang, Q.; Lin, M.; Yang, M. P (VPBA-DMAEA) as a $\mathrm{pH}$-sensitive nanovalve for mesoporous silica nanoparticles based controlled release. Chin. Chem. Lett. 2015, 26, 1203-1208. [CrossRef]

11. Feng, J.; Wen, W.; Jia, Y.G.; Liu, S.; Guo, J. pH-Responsive Micelles Assembled by Three-Armed Degradable Block Copolymers with a Cholic Acid Core for Drug Controlled-Release. Polymers 2019, 11, 511. [CrossRef] [PubMed]

12. Zhang, C.Y.; Wu, W.S.; Yao, N.; Zhao, B.; Zhang, L.J. pH-sensitive amphiphilic copolymer brush Chol- $g$-P(HEMA-co-DEAEMA)- $b$-PPEGMA: Synthesis and self-assembled micelles for controlled anti-cancer drug release. RSC Adv. 2014, 4, 40232-40240. [CrossRef]

13. Zhao, Z.; Zhu, F.; Qu, X.; Wu, Q.; Wang, Q.; Zhang, G.; Liang, F. pH-Responsive polymeric Janus containers for controlled drug delivery. Polym. Chem. 2015, 6, 4144-4153. [CrossRef]

14. Zhou, J.; Zhang, W.; Hong, C.; Pan, C. Silica Nanotubes Decorated by pH-Responsive Diblock Copolymers for Controlled Drug Release. ACS Appl. Mater. Interfaces 2015, 7, 3618-3625. [CrossRef]

15. Yan, W.; Fantin, M.; Spencer, N.D.; Matyjaszewski, K.; Benetti, E.M. Translating Surface-Initiated Atom Transfer Radical Polymerization into Technology: The Mechanism of $\mathrm{Cu}^{0}$-Mediated SI-ATRP under Environmental Conditions. ACS Macro Lett. 2019, 8, 865-870. [CrossRef]

16. Wang, L.P.; Li, Y.C.; Chen, L.F.; Ban, C.L.; Li, G.; Ni, J.J. Fabrication of honeycomb-patterned porous films from PS-b-PNIPAM amphiphilic diblock copolymers synthesized via RITP. J. Colloid Interface Sci. 2014, 420, 112-118. [CrossRef]

17. Sato, T.; Dunderdale, G.J.; Urata, C.; Hozumi, A. Sol-Gel Preparation of Initiator Layers for Surface-Initiated ATRP: Large-Scale Formation of Polymer Brushes Is Not a Dream. Macromolecules 2018, 51, 10065-10073. [CrossRef]

18. Kang, H.; Jeong, W.; Hong, D. Antifouling Surface Coating Using Droplet-Based SI-ARGET ATRP of Carboxybetaine under Open-Air Conditions. Langmuir 2019, 35, 7744-7750. [CrossRef]

19. Yan, C.N.; Liu, Q.; Xu, L.; Bai, L.P.; Wang, L.P.; Li, G. Photoinduced Metal-Free Surface Initiated ATRP from Hollow Spheres Surface. Polymers 2019, 11, 599. [CrossRef]

20. Yan, W.; Fantin, M.; Ramakrishna, S.; Spencer, N.D.; Matyjaszewski, K.; Benetti, E.M. Growing Polymer Brushes from a Variety of Substrates under Ambient Conditions by $\mathrm{Cu}^{0}$-Mediated Surface-Initiated ATRP. ACS Appl. Mater. Interfaces 2019, 11, 27470-27477. [CrossRef]

21. Discekici, E.H.; Anastasaki, A.; Alaniz, J.R.; Hawker, C.J. Evolution and Future Directions of Metal-Free Atom Transfer Radical Polymerization. Macromolecules 2018, 51, 7421-7434. [CrossRef]

22. Ma, A.; Zhang, J.; Wang, N.; Bai, L.; Chen, H.; Wang, W.; Yang, H.; Yang, L.; Niu, Y.; Wei, D. Surface-Initiated Metal-Free Photoinduced ATRP of 4-Vinylpyridine from $\mathrm{SiO}_{2}$ via Visible Light Photocatalysis for Self-Healing Hydrogels. Ind. Eng. Chem. Res. 2018, 57, 17417-17429. [CrossRef]

23. Kaßel, M.; Gerke, J.; Ley, A.; Vana, P. Surface Modification of Wood Flour via ARGET ATRP and Its Application as Filler in Thermoplastics. Polymers 2018, 10, 354. [CrossRef] [PubMed] 
24. Treat, N.J.; Sprafke, H.; Kramer, J.W.; Clark, P.G.; Barton, B.E.; Alaniz, J.R.; Fors, B.P.; Hawker, C.J. Metal-free atom transfer radical polymerization. J. Am. Chem. Soc. 2014, 136, 16096-16101. [CrossRef]

25. Zeng, G.; Liu, M.; Shi, K.; Heng, C.; Mao, L.; Wan, Q.; Huang, H.; Deng, F.; Zhang, X.; Wei, Y. Surface modification of nanodiamond through metal free atomtransfer radical polymerization. Appl. Surf. Sci. 2016, 390, 710-717. [CrossRef]

26. Ma, L.; Li, N.; Zhu, J.; Chen, X. Visible Light-Induced Metal Free Surface Initiated Atom Transfer Radical Polymerization of Methyl Methacrylate on SBA-15. Polymers 2017, 9, 58. [CrossRef]

27. Yan, J.; Pan, X.; Schmitt, M.; Wang, Z.; Bockstaller, M.R.; Matyjaszewski, K. Enhancing initiation efficiency in metal-free surface-initiated atom transfer radical polymerization (SI-ATRP). ACS Macro Lett. 2016, 5, 661-665. [CrossRef]

28. George, D.; Maheswari, P.U.; Begum, K.M.M.S. Synergic formulation of onion peel quercetin loaded chitosan-cellulose hydrogel withgreen zincoxide nanoparticles towards controlled release, biocompatibility, antimicrobial and anticancer activity. Int. J. Biol. Macromol. 2019, 132, 784-794. [CrossRef]

29. Yan, C.N.; Xu, L.; Liu, Q.D.; Zhang, W.; Jia, R.; Liu, C.Z.; Wang, S.S.; Wang, L.P.; Li, G. Surface-Induced ARGET ATRP for Silicon Nanoparticles with Fluorescent Polymer Brushes. Polymers 2019, 11, 1228. [CrossRef]

30. Trendafilova, I.; Szegedi, A.; Mihály, J.; Momekov, G.; Lihareva, N.; Popova, M. Preparation of efficient quercetin delivery system on Zn-modified mesoporous SBA-15 silica carrier. Mater. Sci. Eng. 2017, 73, 285-292. [CrossRef]

31. Popova, M.; Trendafilova, I.; Tsacheva, I.; Mitova, V.; Kyulavska, M.; Koseva, N.; Mihály, J.; Momekova, D.; Momekov, G.; Aleksandrov, H.A.; et al. Amino-modified KIT-6 mesoporous silica/polymer composites for quercetin delivery: Experimental and theoretical approaches. Microporous Mesoporous Mater. 2018, 270, 40-47. [CrossRef]

32. Yuan, L.; Tang, Q.; Yang, D.; Zhang, J.Z.; Zhang, F.; Hu, J. Preparation of pH-Responsive Mesoporous Silica Nanoparticles and Their Application in Controlled Drug Delivery. J. Phys. Chem. C 2011, 115, 9926-9932. [CrossRef]

33. Zhao, Y.; Cai, C.; Liu, M.; Zhao, Y.; Pei, W.; Chu, X.; Zhang, H.; Wang, Z.; Han, J. An organic solvent-free technology for the fabrication of albumin-based paclitaxel nanoparticles for effective cancer therapy. Colloid Surfaces B 2019, 183, 110394. [CrossRef] [PubMed]

34. Guo, Q.; Liu, M.; Zhao, Y.; Wu, Y.; Liu, J.; Cai, C.; Shi, Y.; Han, J. Spectroscopic and cytotoxicity studies on the combined interaction of (-)-epigallocatechin-3-gallate and anthracycline drugs with human serum albumin. Spectrochim. Acta Part A 2019, 222, 117213. [CrossRef] [PubMed]

35. Fan, Z.; Cheng, P.; Liu, M.; Li, D.; Liu, G.; Zhao, Y.; Ding, Z.; Chen, F.; Wang, B.; Tan, X.; et al. Poly (glutamic acid) hydrogels crosslinked via native chemical ligation. New J. Chem. 2017, 41, 8656-8662. [CrossRef]

36. Arellano-Archán, E.; Alcalá, M.E.; Vega-Becerra, O.E.; Lara-Ceniceros, T.E.; Bonilla-Cruz, J. Optimizing the Coverage Density of Functional Groups over $\mathrm{SiO}_{2}$ Nanoparticles: Toward High-Resistant and Low-Friction Hybrid Powder Coatings. ACS Omega 2018, 3, 16934-16944. [CrossRef]

(C) 2019 by the authors. Licensee MDPI, Basel, Switzerland. This article is an open access article distributed under the terms and conditions of the Creative Commons Attribution (CC BY) license (http://creativecommons.org/licenses/by/4.0/). 